\title{
Determinantes de los precios reales del petróleo y su impacto sobre las principales variables macroeconómicas: EU, España, Noruega y Argentina*
}

Luis N. Lanteri**

\section{RESUMEN}

En este trabajo se analizan los principales determinantes macroeconómicos de los precios reales del petróleo (crecimiento mundial, política monetaria, tipo de cambio del dólar). Asimismo, se evalúan empíricamente el impacto de los cambios en los precios del petróleo sobre la actividad económica, el empleo y los precios domésticos en algunos países importadores netos (Estados Unidos y España), exportadores (Noruega) y autosuficientes en materia petrolífera (Argentina). El trabajo emplea modelos SVAR, con restricciones de corto plazo, para un periodo que abarca las últimas cuatro décadas. Los resultados muestran que, para los países importadores, los efectos de los incrementos de precios sobre el crecimiento del PIB real y el empleo difieren de los efectos que generan las caídas de los precios del crudo.

Palabras clave: precios reales del petróleo, determinantes macroeconómicos, actividad económica y empleo, SVAR, modelos no lineales.

Clasificación JEL: E32, Q43.

\begin{abstract}
This paper analyzes the main macroeconomic determinants of real oil prices (global growth, monetary policy, dollar exchange rate). It also assesses empirically the effects of oil prices shocks on economic activity, employ and domestic prices of some importing countries (United States and Spain), exporters (Norway) and self-sufficient in energy (Argentina). The paper uses SVAR models, with short-term restrictions, covering a period spanning the last four decades. The results show that, for importing countries, the effects of an increase in oil prices on real GDP growth and employment differ from those of an oil price decrease.
\end{abstract}

Keywords: real oil prices, macroeconomic determinants, economic activity and employment, SVAR, non-lineal models.

JEL classification: E32, Q43.

* Fecha de recepción: 21/09/2012,. Fecha de aprobación: 05/09/2014.

** Investigador independiente, Argentina. Correo electrónico: lnlante@yahoo.com.ar. 


\section{INTRODUCCIÓN}

En los últimos años se ha observado un importante incremento en los precios internacionales de las materias primas, tanto de alimentos y minerales, como del petróleo. En el caso del crudo, los precios reales de comienzos de 2011 fueron tres veces más elevados que el promedio de los años noventa, superando además los niveles récord de las cuatro décadas anteriores, con excepción de 2008. Los cambios que tuvieron lugar en los balances de oferta-demanda habrían sido una de las principales causas de las fluctuaciones de precios ocurridas en este último periodo.

En el caso de algunas de las principales materias primas, la expansión de la oferta resultó insuficiente para satisfacer el crecimiento de la demanda mundial, lo que afectó los inventarios e hizo más sensibles estos mercados a diferentes impactos exógenos. La rápida expansión de las actividades manufactureras en las economías emergentes, combinada con la creciente urbanización y los cambios en la conducta de los consumidores, habría empujado la demanda de alimentos, metales y, también, de energía, en particular petróleo (IMF, 2011b, pp. 101-133; IMF, 2012, pp. 125-169).

Para Nakaso (2011), el fuerte crecimiento global habría sido el principal impulsor de los precios de las materias primas. A su vez, la existencia de una política monetaria acomodaticia por parte de los bancos centrales de las economías avanzadas y, en menor medida, la participación de inversores financieros y especuladores en estos mercados (Frankel, 2008; Kilian y Hicks, 2009; Singleton, 2011), habrían contribuido también a los cambios en la demanda agregada y a las fluctuaciones en los precios internacionales de las materias primas, particularmente en el periodo posterior a la crisis financiera de 2008.

Asimismo, varios trabajos, entre ellos los de Hamilton (1983), Gisser y Goodwin (1986), Bjornland (2000), Cuñado y Pérez de Gracia (2003), Hamilton (2003) y Elder y Serletis (2010), encuentran que los incrementos en los precios del petróleo habrían afectado negativamente a la economía mundial (al constituir una de las fuentes de las fluctuaciones macroeconómicas). De acuerdo con Hamilton (2009), los incrementos en los precios del petróleo habrían sido los causantes de la mayor parte de las recesiones observadas en Estados Unidos (Eu), desde finales de la Segunda Guerra Mundial, y habrían tenido consecuencias negativas para los países importadores de recursos petrolíferos. Para los países exportadores, en cambio, los aumentos de precios podrían llegar a ser significativamente diferentes. Por lo general, precios más elevados implicarían 
mayores ingresos y riqueza para estas economías y repercutirían favorablemente sobre sus niveles de actividad. ${ }^{1}$

En este trabajo se intenta establecer los principales determinantes macroeconómicos que afectan la variabilidad de los precios reales del petróleo y analizar, a su vez, el impacto de los cambios de precio del crudo sobre la actividad económica, el empleo y los precios domésticos en algunos países importadores netos (EU y España), exportadores (Noruega) o autosuficientes (Argentina).

En la sección I, se analiza el mercado mundial y los precios reales del petróleo y en la II se muestra la metodología utilizada y los supuestos y restricciones impuestas a los modelos vectoriales autorregresivos estructurales (SVAR, por sus siglas en inglés: structural vector autoregressive) utilizados en el estudio. A su vez, en la sección III se presentan los resultados de las estimaciones y finalmente se comentan las principales conclusiones.

\section{MERCADO MUNDIAL Y PRECIOS REALES DEL PETRóLEO}

De los datos de producción y consumo mundial de petróleo correspondientes a 2010 se infiere que en ese año hubo un déficit de 5287000 de barriles diarios, que debería haberse cubierto principalmente a partir de los inventarios acumulados en el pasado (cuadro A1.1 de los anexos).

Entre los grandes productores, se destacan la Federación Rusa (10270 000 de barriles diarios) y Arabia Saudita (10 007000 de barriles diarios). Este último país también posee, junto con Venezuela, las mayores reservas comprobadas. Las de Rusia, cuyas exportaciones dependen principalmente del combustible y sus derivados (además de gas), representarían menos de un tercio de las de Arabia Saudita. Otros países con importantes reservas comprobadas son Irán, Irak, Kuwait, Emiratos Árabes, Libia, Kazajistán y Nigeria.

EU (19 148000 de barriles diarios) y China (9057000) encabezan la lista de mayores consumidores de petróleo, seguidos por Japón (4451000), India (3319000), Federación Rusa (3 199000), Arabia Saudita (2812000), Brasil

\footnotetext{
${ }^{1}$ Sin embargo, los trabajos empíricos difieren al evaluar los efectos de los cambios en los precios del crudo sobre el desempeño macroeconómico de los países exportadores de petróleo. Mientras que Noruega (una economía exportadora neta) se habría visto beneficiada por los precios más elevados del petróleo al registrar mayores tasas de crecimiento y menor desempleo, otras economías exportadoras, como Canadá y el Reino Unido, habrían tenido un comportamiento más en línea con el de los países importadores, mostrando una declinación en las tasas de crecimiento (Bjornland, 2000; Jiménez-Rodríguez y Sánchez, 2005).
} 
(2604000), Alemania (2441000), Corea del Sur (2384000) y Canadá (2276000), entre otros grandes demandantes mundiales.

En este contexto, EU sería considerado un país importador neto de petróleo (aunque sigue siendo todavía un importante productor mundial, su producción se redujo a la mitad desde los años setenta), lo mismo que España, mientras que Noruega sería un exportador neto gracias a los hallazgos de crudo en la plataforma submarina del Mar del Norte, a comienzos de los setenta (pese a que su explotación declina desde hace una década). No obstante, cabe destacar que EU ha aumentado considerablemente su producción en los últimos años merced a la aplicación de nuevos sistemas de extracción no convencionales. Por su parte, la Argentina logró el autoabastecimiento en los años noventa, pero por no haber seguido una política coherente en la materia ha venido reduciendo sus reservas de petróleo en la última década y estaría muy cerca de pasar nuevamente a ser importador neto; no obstante, las estadísticas compiladas por British Petroleum (2011) señalan un saldo positivo en el balance de crudo de este país para 2010. Argentina contaría también con grandes reservas de hidrocarburos no convencionales en la Patagonia (yacimiento de Vaca Muerta), aunque todavía no han comenzado a explotarse en forma comercial.

Cuadro 1. Precios reales del petróleo $\left(1970_{1}-2011_{2}\right)$ *

\begin{tabular}{ccc}
\hline & WTI real & Brent real \\
$1970-1979$ & 0.29 & 0.31 \\
$1980-1989$ & 0.46 & 0.45 \\
$1990-1999$ & 0.25 & 0.23 \\
$2000-2009$ & 0.50 & 0.49 \\
$2000-2011$ & 0.54 & 0.53 \\
$2011_{1}$ & 0.84 & 0.93 \\
$2011_{2}$ & 0.91 & 1.04 \\
Mínimo histórico & $0.13\left(1973_{2}\right)$ & $0.09\left(1970_{4}\right)$ \\
Máximo histórico & $1.14\left(2008_{2}\right)$ & $1.13\left(2008_{2}\right)$ \\
\hline
\end{tabular}

* Los subíndices indican el trimestre del año correspondiente.

Precios reales: se utilizaron los precios, en dólares estadounidenses, provenientes de las Estadísticas Financieras Internacionales del Fondo Monetario Internacional (IMF, 2011a) y el deflactor del PIB de EU (base $2005=100$ ).

Los precios reales del petróleo, que habían registrado niveles récord a principios de los ochenta, declinaron durante las dos décadas posteriores, hasta 
caer a valores mínimos a finales de los años noventa (cuadro 1). A partir de 2002, se reinicia una tendencia creciente, alcanzando máximos históricos en el año 2008. Luego de la crisis financiera internacional, los precios mostraron una pronunciada caída, pero lograron recuperarse posteriormente, a partir de la reducción en las tasas de interés internacionales, las políticas de estímulo monetario, por parte de la Reserva Federal de Eu y del Banco Central Europeo, y la sostenida demanda de algunas economías emergentes, como China y la India. En este sentido, las cotizaciones en términos reales de principios de 2011 habrían sido las más elevadas desde la década de los setenta, con excepción de las observadas durante el año 2008 (gráfica 1).

Los precios, en dólares estadounidenses, que se consignan del West Texas Intermediate (WTI) y del Brent fueron tomados de los datos compilados en las Estadísticas Financieras Internacionales del Fondo Monetario Internacional (IMF, 2011a). Los precios reales se obtienen al aplicarle a los nominales el deflactor del producto interno bruto (PIB) de EU (base $2005=100$ ). En el primer trimestre de 1970, el precio nominal del WTI era de USD 3.35 por barril y el del Brent de USD 2.23 por barril.

Gráfica 1. Precios reales del petróleo WTI y Brent estimados utilizando el deflactor del PIB de EU

(base $2005=100$ )

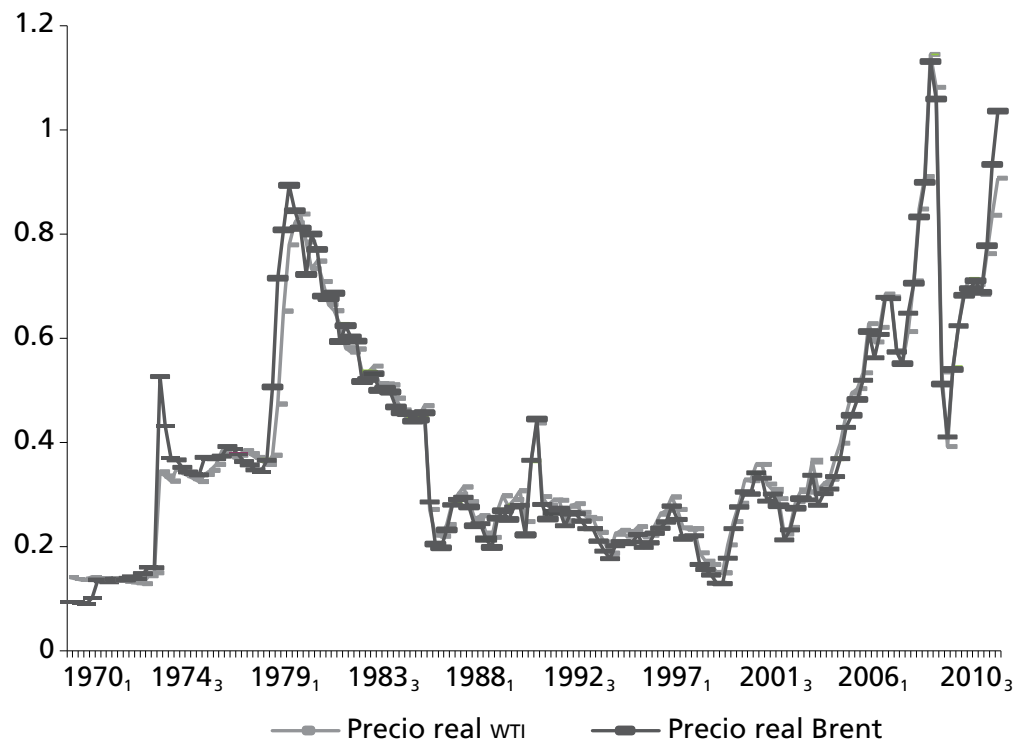


El fuerte crecimiento global, la política monetaria de algunas de las principales economías y la depreciación del dólar frente al resto de las monedas habrían contribuido a los incrementos observados en los precios internacionales de las materias primas. El crecimiento económico de algunas naciones asiáticas favoreció la expansión de la demanda mundial e impulsó los precios de los productos primarios (Trinh, Voss y Dyck, 2006; Cheung y Morin, 2007). No obstante, ello habría tenido lugar, por lo general, en un contexto de abundante liquidez en los mercados internacionales y de aumentos en la oferta insuficientes para satisfacer los requerimientos de una demanda creciente. ${ }^{2}$

Lo ocurrido en los setenta, durante la crisis energética, y más recientemente durante el auge de precios de 2008, cuando las cotizaciones de la mayoría de las materias primas se movieron en la misma dirección, pone de relieve la influencia de los factores macroeconómicos en el estudio de los mercados de materias primas. Entre ellos, pueden mencionarse:

a) El aumento de la demanda mundial que, debido al crecimiento del nivel de actividad en las principales economías, sería uno de los factores que habría impulsado, en los últimos años, los precios reales del petróleo. De acuerdo con el Banco Mundial (Nakaso, 2011), la demanda de petróleo se incrementó alrededor de 25\% durante el periodo 2000-2010. EU, China, Japón, India, Alemania y Corea del Sur serían los principales importadores netos de crudo, seguidos por otros países desarrollados (Francia, España, Italia, Singapur, Holanda, etcétera). ${ }^{3}$ Algunas economías emergentes, como China e India, representaban un porcentaje bastante más reducido de la demanda mundial hasta hace dos décadas. Sin embargo, las compras de China habría estado creciendo a $7 \%$ anual acumulativo desde 1990, mientras que algunas importantes economías avanzadas habrían declinado su demanda en los últimos años (Hamilton, 2009).

b) La política monetaria de las economías desarrolladas, que actuaría a través de varios canales. El canal de las tasas de interés muestra que dicha política influye sobre los precios de los bienes a través de la de-

\footnotetext{
${ }^{2}$ También habría contribuido la baja elasticidad-precio de la demanda de crudo (Hamilton, 2009).

${ }^{3}$ Estimado de acuerdo con la diferencia entre la producción y el consumo de cada país (año 2010). No obstante, parte de esa diferencia podría haberse cubierto con los inventarios formados en el pasado.
} 
manda agregada y de la inflación. ${ }^{4}$ Tasas de interés (reales) bajas estimularían la demanda de todo tipo de bienes, incluyendo las materias primas, y reducirían el costo de oportunidad de mantener inventarios, lo cual afectaría los balances de oferta-demanda en estos mercados. Frankel y Rose (2010) sugieren que cuando las tasas de interés (reales) son elevadas, como en la década de los ochenta, los inversores se desprenderían de sus posiciones en materias primas. Este comportamiento se mantendría hasta que los precios fueran percibidos por debajo del valor de equilibrio de largo plazo, generando expectativas de futuros incrementos de precios (al satisfacer la condición de arbitraje). Por el contrario, cuando las tasas de interés son bajas, como en la última década (2002-2005 y 2008-2011), los inversores se posicionarían en las materias primas. Esta conducta continuaría hasta que los precios sean percibidos por arriba de su valor de equilibrio de largo plazo, generando expectativas de caídas en el futuro (al satisfacer la condición de especulación). Para dichos autores, la especulación habría sido uno de los factores más importantes que explican los incrementos de precios entre 2003 y 2008. Sobre el particular, Anzuini, Lombardi y Pagano (2010) encuentran evidencia empírica respecto del impacto significativo de la política monetaria sobre los precios de las materias primas. Una política monetaria expansiva incrementaría el índice agregado de precios y sus principales componentes. Los resultados serían robustos frente a diferentes estrategias de identificación de las repercusiones de la política monetaria.

c) Los cambios en el valor del dólar respecto de otras monedas, que también impactarían los precios de las materias primas. La relación negativa que existe entre el valor del dólar estadounidense y los precios de las materias primas, expresados en esta divisa, tiene su sustento en la ley de un solo precio para los bienes transables. De acuerdo con este argumento, una depreciación del dólar debería compensarse mediante un incremento en los precios de las materias primas en dólares (y/o una caída de los precios en otras monedas), a efectos de asegurar el mismo importe medido en dólares. Asimismo, dado que la mayor parte de las materias

\footnotetext{
${ }^{4}$ La caída en las tasas de interés incrementa el nivel de actividad y, con ello, la demanda de materias primas. Asimismo, éstas podrían servir como resguardo contra la inflación. De esta forma, un aumento en la tasa de inflación de largo plazo esperada estimularía la demanda de materias primas y el precio real presente (Frankel y Rose, 2010). Otros canales que afectan la demanda debido a cambios en las tasas de interés serían los del crédito y el de los préstamos bancarios.
} 
primas se cotizan en dólares en los mercados internacionales, una depreciación de esta divisa incrementaría el poder de compra y la demanda de materias primas por parte de los consumidores externos y reduciría la oferta de los productores externos debido a la menor rentabilidad resultante en otras monedas (Gilbert, 2010). Asimismo, los cambios en los precios del petróleo podrían afectar la actividad económica y el empleo de los países exportadores e importadores, e impactar, a su vez, en los precios domésticos. Estos serían los principales efectos macroeconómicos resultantes de las fluctuaciones en los precios del crudo.

Para Jiménez-Rodríguez y Sánchez (2005), el impacto de los aumentos de precios sería distinto para las economías exportadoras (good news) que para las importadoras (bad news), y a la inversa cuando las cotizaciones se reducen. Los mecanismos de transmisión a través de los cuales los precios del crudo afectan la actividad económica incluyen canales de oferta y de demanda. Por el lado de la primera, se considera que los aumentos de precios, por ser el petróleo un insumo básico en la producción, implicarían incrementos de costos y caídas en la actividad económica. Por el lado de la segunda, los cambios de precios repercutirían en el consumo (especialmente cuando son percibidos como permanentes), al disminuir el ingreso disponible; y en la inversión, debido a los mayores costos y al encarecimiento de los servicios. No obstante, las fluctuaciones de precios podrían afectar también a los mercados cambiarios y la inflación doméstica, con repercusiones indirectas sobre el nivel de actividad. ${ }^{5}$

Para algunos autores (Blanchard y Galí, 2007), el impacto de los precios del crudo en las economías avanzadas estaría disminuyendo con el paso del tiempo, debido, entre otros factores, a la mayor flexibilidad del mercado laboral, a los cambios en la política monetaria y a que la participación del crudo en la economía podría haber estado declinando desde los setenta. Por ello, los efectos de los cambios en los precios del petróleo sobre el crecimiento, el empleo y la inflación podrían haber sido menores en las dos últimas décadas. ${ }^{6}$

Los aumentos en la cotización del crudo podrían impactar, a su vez, en los costos de la producción de alimentos y en el mercado de biocombustibles, impulsando la demanda de algunas materias primas agrícolas, como el maíz, la

\footnotetext{
${ }^{5}$ Los elevados precios de las materias primas generan presiones inflacionarias y pérdidas en los ingresos reales de los consumidores, en especial en los países importadores de bajos ingresos; al respecto, véase Sachs (1982) y Brown y Yucel (1999).

${ }^{6}$ Estos autores utilizan modelos de vectores autorregresivos en sus estimaciones.
} 
soja y la caña de azúcar (Coyle, 2007; Baffes, 2007), aunque para otros autores esta relación no sería todavía del todo clara.

\section{Metodología UTILIZAda EN LAS ESTIMACIONES}

En el trabajo, se emplean modelos SVAR para establecer los determinantes macroeconómicos que afectan la variabilidad de los precios reales del petróleo y para analizar el impacto de dichos precios sobre la actividad económica, el empleo y los precios domésticos, en los casos de algunos países importadores, exportadores y autosuficientes en materia petrolera.

\section{La propuesta de modelo SVAR con restricciones de corto plazo}

Las estimaciones utilizan modelos SVAR con restricciones de corto plazo en las variables. Si $y_{t}$ es un vector de $k$ variables endógenas, entonces la ecuación estructural del modelo podría representarse como:

$$
A y_{t}=C(L) y_{t}+B \mu_{t}
$$

donde los errores estocásticos estructurales (no observables) $\mu_{t}$ se distribuyen normalmente, es decir, $\mu_{t} \sim N(0, I) ; L$ es el operador de retrasos, y $A, B$ y $C$ son matrices no observables separadamente $(k x k)$. Sin embargo, no es posible estimar directamente la expresión anterior debido a problemas de identificación. En este caso, se recurre a un modelo vectorial autorregresivo (VAR) sin restricciones y se le imponen restricciones a efectos de identificar su estructura subyacente:

$$
y_{t}=A^{-1} C(L) y_{t}+A^{-1} B \mu_{t}
$$

A su vez, podrían estimarse los residuos estocásticos, $A^{-1} B \mu_{t}$, a partir de los residuos observados, $\varepsilon_{t}$, del modelo VAR sin restricciones:

$$
A^{-1} B \mu_{t}=\varepsilon_{t}
$$

Al reformular la ecuación (3): $A^{-1} B \mu_{t} \mu_{t}^{\prime} B^{\prime}\left(A^{-1}\right)^{\prime}=\varepsilon_{t} \varepsilon_{t}^{\prime}$ y tomando la esperanza matemática: $A^{-1} B E\left[\mu_{t} \mu_{t}^{\prime}\right] B^{\prime}\left(A^{-1}\right)^{\prime}=E\left[\varepsilon_{t} \varepsilon_{t}^{\prime}\right]$, y siendo $\Sigma=E\left[\varepsilon_{t} \varepsilon_{t}^{\prime}\right]$ la matriz de varianza-covarianza y $E\left[\mu_{t} \mu_{t}^{\prime}\right]=I$, se obtiene 


$$
A^{-1} B B^{\prime}\left(A^{-1}\right)^{\prime}=\Sigma
$$

donde $\varepsilon_{t}$ y $\mu_{t}$ son vectores de dimensión $k$. Por tanto,

$$
A \Sigma A^{\prime}=B B^{\prime}
$$

Para un modelo con $k$ variables, las propiedades de simetría determinan que deban imponerse $[k(3 k-1)] / 2$ restricciones adicionales. Amisano y Giannini (1997) plantean que el esquema de restricciones tomaría la siguiente forma:

$$
A \varepsilon_{t}=B \mu_{t}
$$

\section{Supuestos y restricciones a imponer a los modelos SVAR}

Para analizar la importancia de los diferentes determinantes macroeconómicos de los precios reales del petróleo se consideran cuatro variables endógenas: 1) el índice de producción industrial de las economías avanzadas (prodind) $;^{7} 2$ ) la tasa de interés real de corto plazo de EU, en la que se emplea el deflactor del PIB de ese país, con datos interanuales (irealus); 3) el tipo de cambio efectivo del dólar estadounidense (neer), para el cual un incremento del índice indicaría una apreciación y viceversa; 4) el precio real del crudo WTI, que se obtiene utilizando también el deflactor del PIB de EU (base $2005=100$ ).

El vector de variables $Y_{t}$ sería el siguiente:

$$
Y_{t}=[\text { prodind, irealus, neer, precio real del WTI }]
$$

Los impactos se identifican imponiendo una estructura causal recursiva sugerida por Sims (1980). Este esquema establece que la primera variable del sistema no reacciona simultáneamente a los impactos en las demás variables, pero que las restantes si lo harían ante los impactos en la primera de manera consecutiva (la tercera reaccionaría en el corto plazo a los impactos en la primera y en la segunda, etcétera). Estas restricciones hacen referencia solamente a relaciones contemporáneas, de forma que después de un periodo trimestral las variables podrían verse afectadas por todos los impactos del sistema.

\footnotetext{
${ }^{7}$ Los datos utilizados en las estimaciones provienen de IMF (2011a). Lamentablemente, no se dispone de una serie de crecimiento de la economía mundial con periodicidad trimestral para el periodo analizado.
} 
Las restricciones (recursivas) sobre los parámetros estructurales contemporáneos serían:

a) El índice de producción industrial no resulta simultáneamente afectado por el resto de los impactos. En el corto plazo respondería solamente a sus propias innovaciones.

b) Las tasas de interés de EU responden de manera sincrónica a sus propios impactos y a las innovaciones en el índice de producción industrial de las economías avanzadas (que incluye la producción de ese país).

c) El tipo de cambio efectivo del dólar resulta contemporáneamente afectado por los impactos en el índice de producción industrial y en las tasas de interés, y por sus propias innovaciones. Este tipo de ordenamiento (primero el nivel de actividad y luego las tasas de interés y el tipo de cambio) es compatible con el trabajo de Eichenbaum y Evans (1995), entre otros.

d) Los cambios en los precios reales del petróleo no afectan a ninguna de las variables en el corto plazo, pero serían influenciados contemporáneamente por todas ellas.

Para Akram (2009), los estudios sobre funciones de reacción de la política monetaria en economías de la Organización para la Cooperación y el Desarrollo Económicos (OCDE) no muestran una respuesta importante de las tasas de interés frente al tipo de cambio, o frente a los cambios de los precios de las materias primas en el corto plazo. Por otra parte, si bien existe evidencia de que el tipo de cambio podría responder a éstos en el largo plazo, los resultados sobre una relación contemporánea entre dichas variables no son tan concluyentes (Cashin, Céspedes y Sahay, 2004).

Debido a las limitaciones en la disponibilidad de información, no es posible incorporar una variable que represente la producción industrial (actividad económica) de China, durante todo el periodo analizado. No obstante, el comportamiento de las economías emergentes (como las de ese país e India) podría estar siendo captado indirectamente a través del índice de producción industrial que da cuenta del crecimiento de las economías avanzadas, dado el proceso de globalización internacional.

A partir de las Estadísticas Financieras Internacionales del FMI (IMF, 2011a) tampoco es posible obtener una serie completa de las exportaciones e importaciones de China, la que podría servir para medir indirectamente la evolución del 
nivel de actividad industrial de ese país. Sobre la base del esquema planteado por Amisano y Giannini (1997), las restricciones indicadas arriba (al especificar $B$ como una matriz diagonal y aplicar en forma recursiva las restricciones sobre la matriz $A$ ) podrían formalizarse como ${ }^{8}$

$$
\left[\begin{array}{cccc}
1 & 0 & 0 & 0 \\
a_{21} & 1 & 0 & 0 \\
a_{31} & a_{32} & 1 & 0 \\
a_{41} & a_{42} & a_{43} & 1
\end{array}\right] *\left[\begin{array}{c}
\varepsilon_{1} \\
\varepsilon_{2} \\
\varepsilon_{3} \\
\varepsilon_{4}
\end{array}\right]=\left[\begin{array}{cccc}
b_{11} & 0 & 0 & 0 \\
0 & b_{22} & 0 & 0 \\
0 & 0 & b_{33} & 0 \\
0 & 0 & 0 & b_{44}
\end{array}\right] *\left[\begin{array}{l}
\mu_{1} \\
\mu_{2} \\
\mu_{3} \\
\mu_{4}
\end{array}\right]
$$

En la propuesta de modelo SVAR, el procedimiento a seguir es el siguiente: En primer lugar, sería necesario estimar la forma reducida de un sistema VAR con $k$ variables endógenas. Luego, deberían imponérsele las restricciones, para que el modelo quede perfectamente identificado y puedan obtenerse las respuestas de corto plazo de las variables a los diferentes impactos estructurales.

También se utiliza la metodología de modelos SVAR con restricciones contemporáneas para establecer los efectos de los cambios en los precios reales del petróleo sobre la actividad económica, el empleo y los precios domésticos de las cuatro economías analizadas. Los modelos serían similares a los planteados en la ecuación (8), con sendas variables endógenas. En este caso, el ordenamiento sería el siguiente:

$$
Y_{t}=[\text { precio real del crudo, PIB real, empleo, precios domésticos }]
$$

Para los EU y Argentina se consideran los precios reales del WTI y para España y Noruega, los del Brent (se supone que, por la proximidad geográfica, son los que se toman como referencia en dichos países). En este caso, se utilizan datos del PIB real, empleo en miles de personas y precios domésticos de acuerdo con el deflactor del PIB de cada país (en el caso de Argentina corresponde al índice de precios al consumidor hasta 2007 y al deflactor del PIB, base $1993=100$,

\footnotetext{
${ }^{8}$ En un modelo con cuatro variables endógenas $(k=4)$, el número de restricciones adicionales a aplicar sobre las matrices $A$ y $B$, para su exacta identificación, sería igual a $22[(k(3 k-1)) / 2]$. Dado que se considera a $B$ como una matriz diagonal con 12 restricciones iguales a cero, las restricciones adicionales a la matriz $A$ serían 10. Ello se cumple con cuatro restricciones iguales a la unidad y seis restricciones iguales a cero $[k(k-1) / 2]$.
} 
después de ese año). Los datos provienen mayormente de las Estadísticas Financieras Internacionales del FMI (IMF, 2011a).

Algunos de los trabajos empíricos que analizan la relación entre los precios del petróleo y la actividad económica utilizan modelos lineales. En este trabajo, se emplean también modelos no lineales de tipo asimétrico en los casos de EU y España, de forma que se puedan captar los efectos de las variaciones positivas (incrementos) y negativas (caídas) de la misma magnitud en los precios reales del petróleo sobre las variables macroeconómicas. La especificación asimétrica, en las cuales las variaciones positivas $\left(O_{t}^{+}\right)$y negativas $\left(O_{t}^{-}\right)$en los precios reales del crudo, serían consideradas variables a incluir en dos modelos diferentes, se define de la siguiente forma (Jiménez-Rodríguez y Sánchez, 2005):

$$
\begin{aligned}
& O_{t}^{+}=O_{t} \text { si } O_{t}>0 \\
& 0 \text { en otro caso } \\
& O_{t}^{-}=O_{t} \text { si } O_{t}<0 \\
& 0 \text { en otro caso. }
\end{aligned}
$$

Las estimaciones se llevan a cabo con las variables en primeras diferencias del logaritmo, salvo las tasas de interés, que se incluyen en primeras diferencias, y con cuatro rezagos en diferencias (la periodicidad más uno), con excepción de las estimaciones que consideran los efectos de los cambios en los precios reales del crudo sobre las variables macroeconómicas (caso de EU para los movimientos positivos y Argentina), para los cuales se incluyen cinco rezagos en diferencias, a fin de evitar autocorrelación en los residuos. Debido a la disponibilidad de información, los modelos abarcan los trimestres 1970 - $2011_{2}$ en el caso de los EU y 1980 -2011 en el de España, Noruega y Argentina.

El índice de producción industrial de las economías avanzadas, el tipo de cambio efectivo del dólar, el deflactor del PIB de EU, el PIB real de Noruega y el PIB real de Argentina corresponden a datos sin estacionalidad. Cabe agregar que se aplicó la prueba de Dickey-Fuller aumentada para determinar la existencia de raíz unitaria en las variables. Adicionalmente se emplearon también pruebas de raíz unitaria con cambio estructural (Lütkepohl, 1991), las que se incluyen en el cuadro A3.1 de los anexos. En casi todas las series no es posible rechazar la hipótesis nula de existencia de raíz unitaria (al 5\%), al considerar un posible cambio estructural (break) de acuerdo con las fechas señaladas en dicho cuadro. A partir de estos resultados, todas las variables se consideran integradas de orden 
uno, $I(1)$, y se incluyen en las estimaciones en primeras diferencias del logaritmo (las tasas de interés en términos reales en primeras diferencias). Cabe agregar que las pruebas de traza y de máximo valor propio sugieren que las variables del modelo, que estima los determinantes de los precios reales del petróleo, no estarían cointegradas. ${ }^{9}$

\section{RESULTADOS DE LAS ESTIMACIONES}

\section{Determinantes macroeconómicos de los precios reales del petróleo}

Los modelos SVAR permiten estimar las funciones de respuesta de los precios reales del petróleo frente a los impactos de sus principales determinantes macroeconómicos (índice de producción industrial de las economías avanzadas, tasas de interés de EU y tipo de cambio efectivo de dólar), ${ }^{10}$ así como establecer la importancia de cada uno de estos impactos en la varianza de los precios reales del crudo (importancia relativa de cada innovación aleatoria).

Gráfica 2. Respuestas acumuladas del precio real del crudo WTI frente al impacto de un desvío estándar en sus principales determinantes $\left(1970_{1}-2011_{2}\right)$

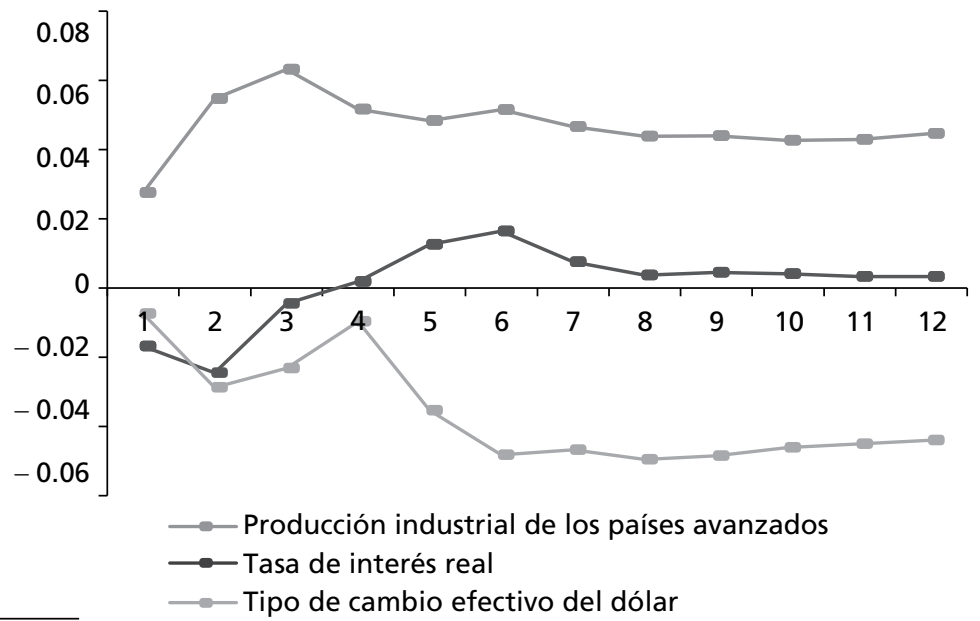

\footnotetext{
${ }^{9}$ Se empleó el programa EViews 8 para la prueba de Dickey-Fuller aumentada y las estimaciones econométricas y el JMulTi para las pruebas de raíz unitaria con cambio estructural. Están a disposición de los lectores los datos empleados en el trabajo y en las estimaciones.

${ }^{10}$ Las funciones de impulso-respuesta corresponden a la descomposición estructural (impactos de un desvío estándar). Por razones de espacio, se excluye la respuesta a su propio impacto. Se consideran las respuestas acumuladas a efectos de recuperar su nivel.
} 
En la gráfica 2 se observa que los precios reales del crudo responden positiva y permanentemente frente a los impactos del índice de producción industrial de las economías avanzadas y en forma negativa y transitoria frente a los de las tasas de interés reales. Por su parte, las innovaciones en el tipo de cambio efectivo del dólar afectan negativa y permanentemente a los precios reales del petróleo. Así, las mejoras en el crecimiento mundial favorecerían un aumento en los precios reales del crudo, mientras que mayores tasas de interés y un dólar más apreciado inducirían caídas, por lo menos en el corto plazo.

Los resultados del análisis de varianza, al especificar un modelo SVAR con las variables en niveles, como en el trabajo de Akram (2009), ${ }^{11}$ muestran que en el corto plazo (primer periodo) la variabilidad de los precios reales del petróleo se explica principalmente por sus propios impactos (cerca de 93\%), mientras que después de transcurridos 36 trimestres, los impactos provenientes de la producción industrial de las economías avanzadas explican 9.5\% y los de las tasas de interés reales y del tipo de cambio efectivo del dólar, lo hacen en $22 \%$, en cada variable. Asimismo, el análisis de varianza muestra que los impactos de las tasas de interés y del tipo de cambio del dólar habrían sido más importantes para explicar la variabilidad de los precios reales del petróleo que el crecimiento de las economías avanzadas (1970-2011).

\section{Efectos de los precios reales del petróleo sobre la actividad económica, el empleo y los precios domésticos}

En las gráficas A2.1, A2.2, A2.3 y A2.4 de los anexos se indican las respuestas de la actividad económica, el empleo y los precios domésticos de cada una de las economías analizadas, frente a cambios en los precios reales del petróleo, de acuerdo con los modelos SVAR especificados con las variables en primeras diferencias del logaritmo. En los casos de Eu y de España (países importadores netos), se indican tanto los movimientos positivos (al alza) de los precios reales (a la izquierda) como los de retroceso (a la derecha). Se observa cómo los primeros generan caídas en los niveles de actividad y en el empleo, aunque en el caso de España esta respuesta negativa se presenta recién después del tercer o cuarto trimestre de iniciado el impacto, mientras que en Eu la respuesta negativa es per-

\footnotetext{
${ }^{11}$ El análisis de varianza estaría alineado así con la estimación de las funciones de respuesta que, al ser acumuladas, vuelven a recuperar el nivel de las variables. Debe notarse que, con el modelo VAR en diferencias, la participación de las variables macroeconómicas en la varianza de los precios reales del crudo sería menor (la mayor parte lo explicaría su propio impacto).
} 
manente. Las caídas de los precios afectan en forma positiva y permanentemente al producto y al empleo de ambos países. Por su parte, los precios domésticos se incrementan tanto frente a los movimientos positivos como negativos de los precios del crudo. Los resultados sugieren que para las economías importadoras netas la relación entre precios y actividad económica (o empleo) sería no lineal.

En los casos de Noruega y Argentina (exportador de petroleo el primero y autosuficiente el segundo), los impactos de los precios del crudo dan lugar a efectos positivos y permanentes en la actividad económica y en el empleo. ${ }^{12}$ No obstante, mientras que, en el primer caso, los precios domésticos muestran una respuesta al alza frente a los impactos de los precios del petróleo, en Argentina la respuesta de los precios internos es a la baja y de forma permanente (ello podría deberse a la existencia de subsidios y a la desvinculación del precio interno del crudo respecto del internacional, debido a las políticas aplicadas por el gobierno).

En todos los modelos que fueron estimados, no se rechaza la hipótesis nula de ausencia de correlación serial en los residuos, de acuerdo con la prueba LM, mientras que las raíces inversas correspondientes al polinomio autorregresivo característico se encuentran dentro del círculo unitario, lo cual sugire que dichas estimaciones serían estables (cuadro A3.2 de los anexos). Asimismo, en el modelo de los determinantes de los precios del petróleo (en el cual se incluye la descomposición de la varianza), no se rechaza la hipótesis nula de no heterocedasticidad, incluyendo términos cruzados (cross terms): $X^{2}=1569.5$, probabili$\mathrm{dad}=0.18$, aunque se rechaza la hipótesis nula de normalidad de los residuos.

En el resto de los modelos, sólo es posible no rechazar la hipótesis nula de no heterocedasticidad en el modelo SVAR que estima los cambios de precios negativos del petróleo para EU (con términos cruzados, $X^{2}=1552$, probabilidad $=0.28)$ y en el modelo para Noruega ( $\sin$ términos cruzados, $X^{2}=343$, probabilidad $=0.18$ ). La hipótesis nula de normalidad de los residuos (conjunta), a través del test de Jarque-Bera, es rechaza en casi todos los casos, salvo en el modelo para Noruega $($ Jarque-Bera $=12.1$, probabilidad $=0.15$ ).

La falta de normalidad podría menoscabar la validez de la inferencia de las estimaciones, aunque el rechazo a la hipótesis de ausencia de heterocedasticidad, dado que los modelos se estiman con las variables en diferencias (estacionarias), sólo afectaría la eficiencia de los estimadores (corresponde al test de heterocedasticidad de White para los residuos; en ciertos casos, al emplear la

\footnotetext{
${ }^{12}$ En Argentina, el empleo se reduce durante el primer periodo de respuesta.
} 
opción con términos cruzados, no resulta posible la estimación con el programa utilizado).

\section{Conclusiones}

En los últimos años se ha registrado un notable incremento en los precios de las materias primas, que ha comprendido tanto a los minerales y los alimentos, como a los recursos energéticos. En el caso del petróleo, los precios reales de principios de 2011 habrían sido los más elevados de las últimas cuatro décadas, con excepción de las cotizaciones observadas en los meses previos a la última crisis financiera internacional.

Para algunos autores (Bjornland, 2000; Jiménez-Rodríguez y Sánchez, 2005; Hamilton, 2009) tales aumentos tendrían consecuencias negativas para las economías importadoras, al verse afectados el consumo y la inversión. Para los países exportadores, en cambio, los incrementos de precios podrían implicar mayores ingresos y riqueza y repercutir favorablemente en la actividad económica y en el empleo de esas economías.

En este trabajo, se analizan los principales determinantes macroeconómicos que afectan la variabilidad de los precios reales del petróleo y se evalúa, a su vez, el impacto de los precios del crudo sobre la actividad económica, el empleo y los precios domésticos de algunos países importadores netos (Estados Unidos y España), exportadores (Noruega) y autosuficientes en abasto petrolífero (Argentina). A tal efecto, se emplean modelos SVAR con restricciones de corto plazo, datos trimestrales que abarcan las últimas cuatro décadas (a partir de 1970 para EU y desde 1980 para las otras tres economías) y variables en primeras diferencias del logaritmo (las tasas de interés en diferencias).

Los resultados de las estimaciones sugieren que los precios reales del crudo responden al alza y de forma permanente frente a los impactos del índice de producción industrial de las economías avanzadas. Por el contrario, tienen una reacción a la baja ante los impactos de las tasas de interés (de manera transitoria) y a los impactos del tipo de cambio efectivo del dólar (de modo permanente). A su vez, el análisis de varianza muestra que en el corto plazo (primer trimestre) la variabilidad de los precios reales del petróleo se explicaría principalmente por sus propios impactos, mientras que después de transcurridos 36 trimestres las tasas de interés reales y el tipo de cambio efectivo del dólar $(22 \%$ cada impacto), así como el índice de producción industrial de las economías 
avanzadas (9.5\%), serían los determinantes macroeconómicos más importantes, con excepción del impacto propio. ${ }^{13}$

Por su parte, los modelos que emplean impactos asimétricos de los precios reales del petróleo sobre las variables macroeconómicas, muestran que los incrementos en los precios del crudo generan caídas en el producto y en el empleo (en EU en forma permanente y en España en el mediano y el largo plazo), mientras que las caídas afectan positivamente la producción y el empleo de ambas economías. De esta forma, se observa para las economías importadoras la existencia de un comportamiento asimétrico frente a las variaciones en los precios reales del petróleo.

En los países exportadores o autosuficientes, los movimientos de precios impactan positivamente en el crecimiento de la actividad económica y en el empleo (modelos simétricos). De la misma manera, los precios domésticos responden en todos los casos al alza respecto a los precios reales del petróleo (EU y España muestran la misma respuesta frente a los movimientos de precios, tanto positivos como negativos), excepto en Argentina, donde se observa una respuesta negativa permanente.

En particular, los resultados de las funciones de respuesta sugieren que los precios reales del crudo podrían incrementarse frente a un mayor crecimiento de la economía mundial (economías avanzadas), políticas monetarias expansivas estadounidenses y una depreciación del dólar en los mercados de divisas. En este sentido, las medidas de alivio cuantitativo a la política monetaria por parte de la Reserva Federal estadounidense, así como las agresivas inyecciones de liquidez al sistema bancario realizadas por el Banco Central Europeo podrían contribuir a generar aumentos en los precios de las materias primas y del petróleo, por lo menos en el corto plazo, con consecuencias perjudiciales, en términos de presiones inflacionarias y caídas en los niveles de actividad y empleo, para aquellos países importadores de crudo. No obstante, es factible que los efectos de los cambios en los precios del petróleo sobre la economía (producto, empleo e inflación) se hayan ido morigerando con el transcurso del tiempo, en contraste con lo ocurrido en los años setenta, tal como fue planteado en el destacado trabajo de Blanchard y Galí (2007).

${ }^{13}$ Para el análisis de varianza de los determinantes macroeconómicos de los precios reales del petróleo, se emplea un modelo de SVAR con las variables en niveles (en lugar de en diferencias), al igual que en el trabajo de Akram (2009). 


\section{Anexo 1}

Cuadro A1.1. Reservas, producción y consumo mundial de petróleo, para países seleccionados

\begin{tabular}{|c|c|c|c|c|}
\hline & $\begin{array}{c}\text { Reservas a fin de } \\
2010 \\
\text { (miles de } \\
\text { millones de } \\
\text { barriles) }\end{array}$ & $\begin{array}{c}\text { Producción de } \\
2010 \\
\text { (miles de barriles } \\
\text { diarios) }\end{array}$ & $\begin{array}{c}\text { Consumo de } 2010 \\
\text { (miles de barriles } \\
\text { diarios) }\end{array}$ & $\begin{array}{c}\text { Saldo = } \\
\text { producción } \\
\text { - consumo } \\
\text { (miles de barriles } \\
\text { diarios) }\end{array}$ \\
\hline Estados Unidos & 30.9 & 7513 & 19148 & -11635 \\
\hline Canadá & 32.1 & 3336 & 2276 & 1060 \\
\hline México & 11.4 & 2958 & 1994 & 964 \\
\hline Argentina & 2.5 & 651 & 557 & 94 \\
\hline Brasil & 14.2 & 2137 & 2604 & -467 \\
\hline Venezuela & 211.2 & 2471 & 765 & 1706 \\
\hline Francia & - & - & 1744 & -1744 \\
\hline Holanda & - & - & 1057 & -1057 \\
\hline Alemania & - & - & 2441 & -2441 \\
\hline España & - & - & 1505 & -1505 \\
\hline Italia & 1.0 & 106 & 1532 & -1426 \\
\hline Kazajistán & 39.8 & 1757 & 262 & 1495 \\
\hline Noruega & 6.7 & 2137 & 239 & 1898 \\
\hline Federación Rusa & 77.4 & 10270 & 3199 & 7071 \\
\hline Reino Unido & 2.8 & 1339 & 1590 & -251 \\
\hline Irán & 137.0 & 4245 & 1799 & 2446 \\
\hline Irak & 115.0 & 2460 & s.d. & s.d. \\
\hline Kuwait & 101.5 & 2508 & 413 & 2095 \\
\hline Qatar & 25.9 & 1569 & 220 & 1349 \\
\hline Arabia Saudita & 264.5 & 10007 & 2812 & 7195 \\
\hline Emiratos Árabes & 97.8 & 2849 & 682 & 2167 \\
\hline Argelia & 12.2 & 1809 & 327 & 1482 \\
\hline Angola & 13.5 & 1851 & s.d. & s.d. \\
\hline Libia & 46.4 & 1659 & s.d. & s.d. \\
\hline Nigeria & 37.2 & 2402 & s.d. & s.d. \\
\hline China & 14.8 & 4071 & 9057 & -4986 \\
\hline India & 9.0 & 826 & 3319 & -2493 \\
\hline Indonesia & 4.2 & 986 & 1304 & -318 \\
\hline Japón & - & - & 4451 & -4451 \\
\hline Singapur & - & - & 1185 & -1185 \\
\hline Corea del sur & - & - & 2384 & -2384 \\
\hline Taiwán & - & - & 1026 & -1026 \\
\hline Tailandia & 0.4 & 334 & 1128 & -794 \\
\hline Total mundial & 1383.2 & 82095 & 87382 & -5287 \\
\hline
\end{tabular}

Fuente: Elaboración propia a partir de datos publicados por British Petroleum (2011). 
Anexo 2. Respuestas acumuladas de la actividad económica (producto), el empleo y los precios domésticos (tasas de inflación) frente a impactos de un desvío estándar en los precios reales del petróleo (EU: trimestres 1970 $-2011_{2}$; otras economías: trimestres $\left.1980_{1}-2011_{2}\right)$.

\section{Gráfica A2.1. Estados Unidos}

Producto

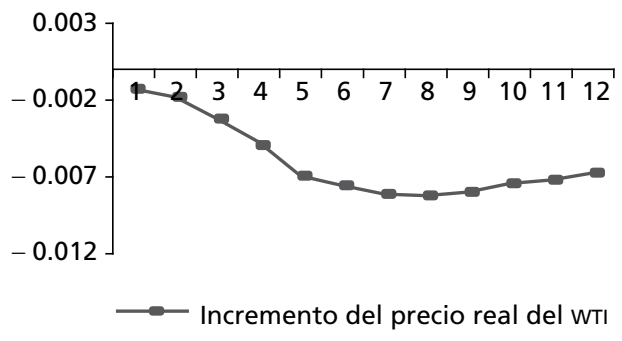

Empleo

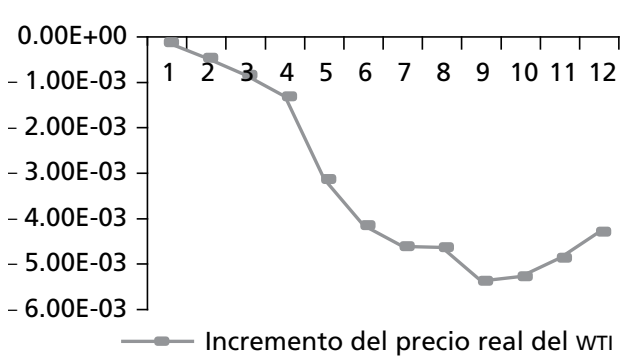

Precios

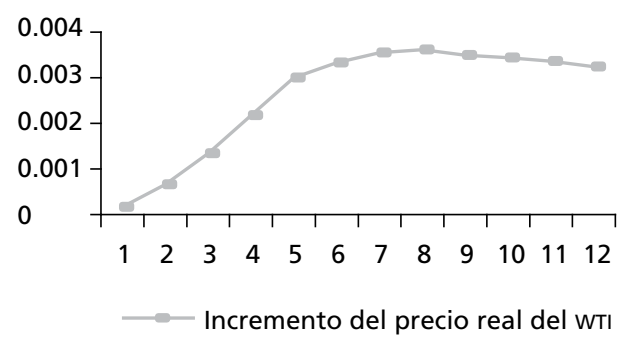

Producto

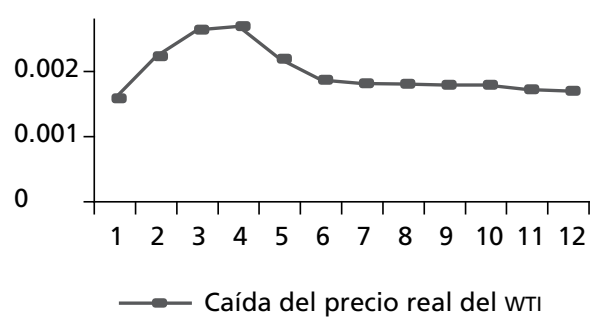

Empleo

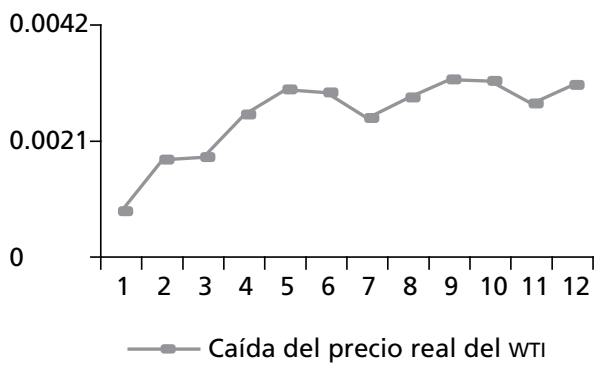

Precios

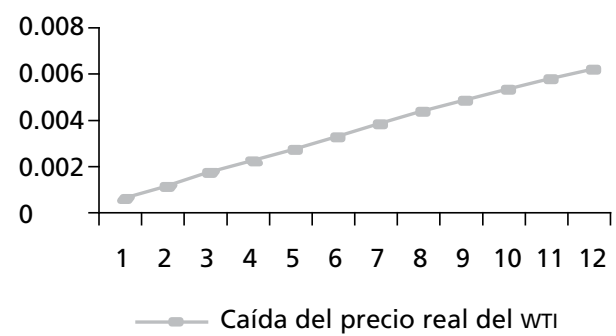


Gráfica A2.2. España

Producto

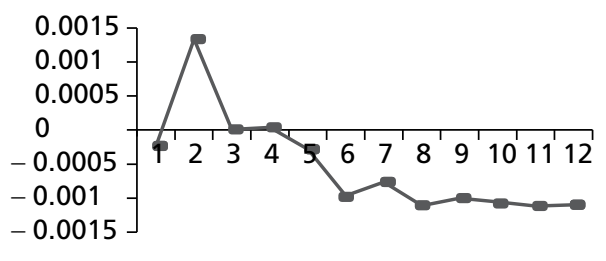

Empleo

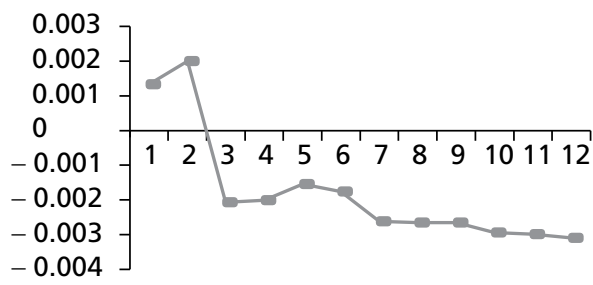

—-Incremento del precio real del Brent

Precios

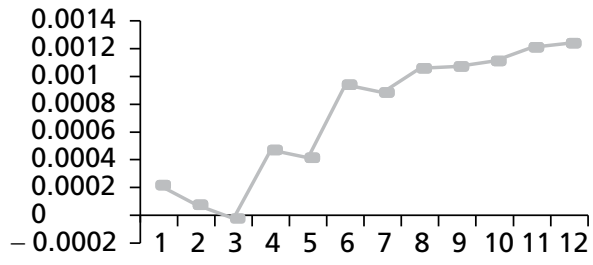

- Incremento del precio real del Brent
Producto

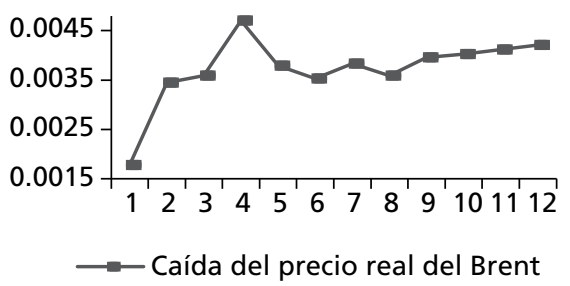

Empleo

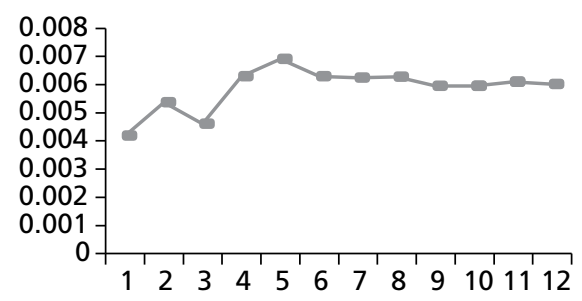

—Caída del precio real del Brent

Precios

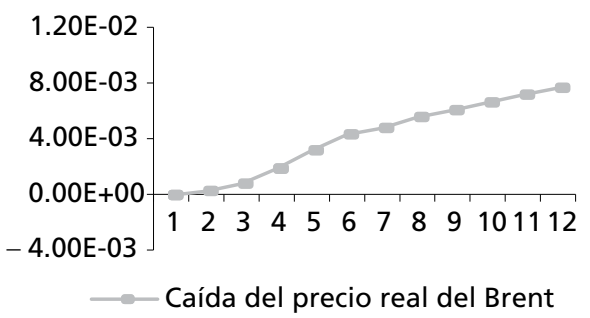

Gráfica A2.3. Noruega (continúa)

Producto

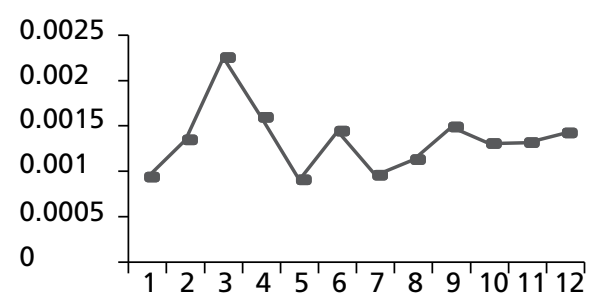

—-Impacto del precio real del Brent
Empleo

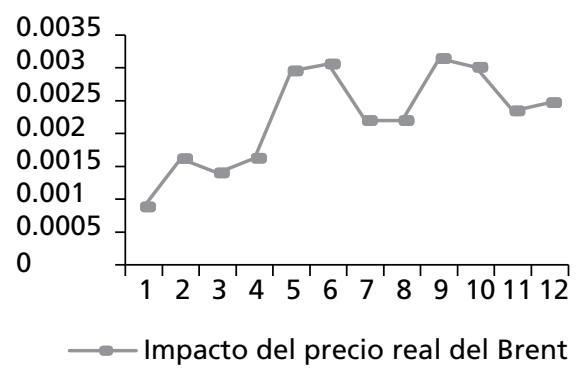


66 ECONOMÍA: TEORÍA Y PRÁCTICA • Nueva Época, número 41, julio-diciembre 2014

Gráfica A2.3. Noruega (concluye)

Precios

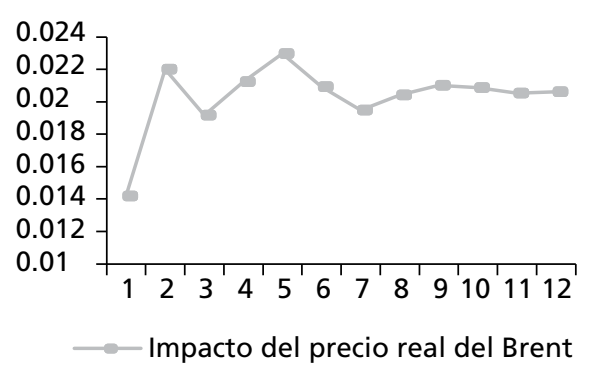

Gráfica A2.4. Argentina

Producto

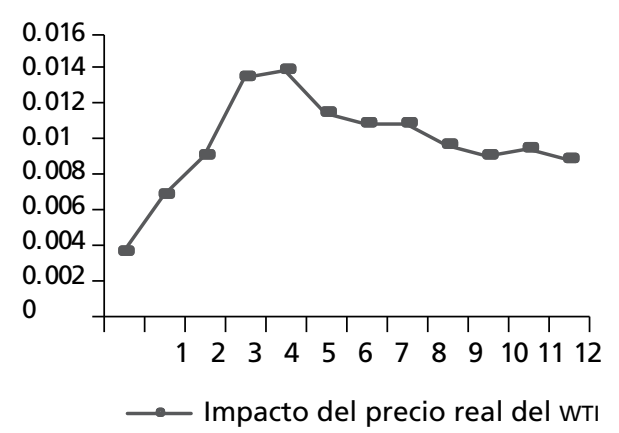

Empleo

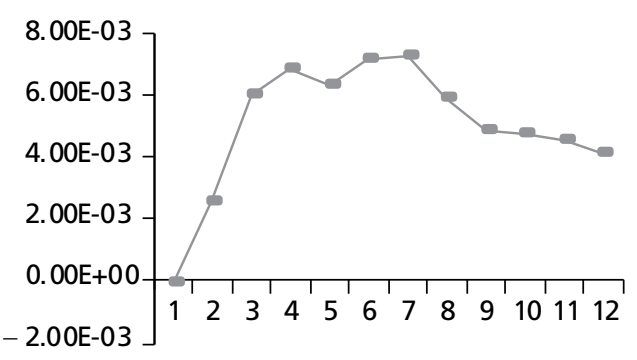

$\ldots$ Impacto del precio real del WTI

\section{Precios}

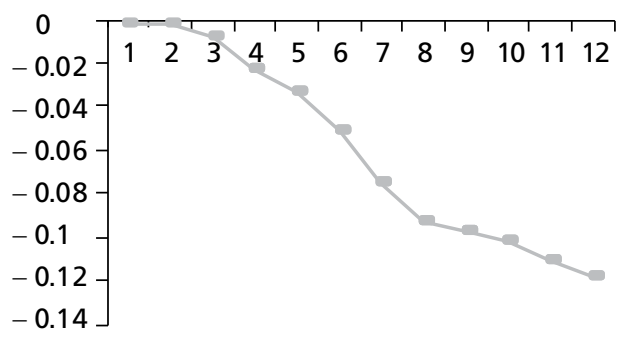

$\ldots$ Impacto del precio real del WTI 


\section{Anexo 3}

Cuadro A3.1. Pruebas de raíz unitaria con cambio estructural

\begin{tabular}{|c|c|c|}
\hline Variable & $\begin{array}{c}\text { Periodo del cambio } \\
\text { estructural (break) por } \\
\text { trimestre }\end{array}$ & Valor de la prueba \\
\hline \multicolumn{3}{|l|}{ Período $1970_{1}-2011_{2}:$} \\
\hline Producción industrial & $2008_{1}$ & -1.57 \\
\hline Tasa de interés real (EU) & $2004_{3}$ & -2.53 \\
\hline Tipo de cambio efectivo (USD) & $2002_{1}$ & -2.20 \\
\hline Precio real del WTI & $2008_{3}$ & -1.72 \\
\hline Precio real del Brent & $2008_{3}$ & -1.78 \\
\hline PIB real (EU) & 2008 & -1.44 \\
\hline Deflactor del PIB (EU) & $1981_{1}$ & -3.34 \\
\hline Empleo (EU) & $2008_{1}$ & -2.66 \\
\hline \multicolumn{3}{|l|}{ Periodo $1980_{1}-2011_{2}$} \\
\hline PIB real (España) & $2008_{3}$ & -1.19 \\
\hline Deflactor del PIB (España) & $1998_{2}$ & -3.06 \\
\hline Empleo (España) & $2005_{2}$ & -1.23 \\
\hline PIB real (Noruega) & $1989_{2}$ & -1.47 \\
\hline Deflactor del PIB (Noruega) & $1998_{4}$ & -0.59 \\
\hline Empleo (Noruega) & $2005_{1}$ & -0.67 \\
\hline PIB real (Argentina) & $2002_{3}$ & 0.92 \\
\hline IPC-deflactor (Argentina) & $1989_{4}$ & -2.67 \\
\hline Empleo (Argentina) & $2002_{3}$ & 0.63 \\
\hline
\end{tabular}

Fuente: Elaboración propia.

Nota: Se emplea la opción shift dummy, con cinco retrasos en niveles (Lütkepohl, 1991). Las ocho primeras variables corresponden al periodo del trimestre $1970_{1}$ al $2011_{2}$ y las restantes al período $1980,-2011_{2}$. En todos los casos no se rechaza la hipótesis nula de existencia de raíz unitaria al $5 \%$ (en el deflactor del PIB de EU y en el de España no se rechaza al 1\%). Para los precios del WTI y del Brent, correspondientes al periodo $1980_{1}-2011_{2}$, se consideraron las mismas fechas para el cambio estructural y tampoco fue posible rechazar la hipótesis nula al $5 \%$. 
68 ECONOMÍA: TEORÍA Y PRÁCTICA • Nueva Época, número 41, julio-diciembre 2014

Cuadro A3.2. Pruebas estadísticas en los modelos SVAR estimados

\begin{tabular}{|c|c|c|c|}
\hline \multirow[t]{2}{*}{ Modelo } & \multicolumn{2}{|c|}{$\begin{array}{c}\text { Test LM de autocorrelación } \\
\text { de residuos }\end{array}$} & \multirow{2}{*}{$\begin{array}{c}\text { Raíz inversa del } \\
\text { polinomio autorregresivo } \\
\text { característico }\end{array}$} \\
\hline & Test & Probabilidad & \\
\hline $\begin{array}{l}\text { Determinantes de los } \\
\text { precios reales del petróleo }\end{array}$ & 25.0 & 0.07 & $\begin{array}{c}\text { Dentro del círculo } \\
\text { unitario }\end{array}$ \\
\hline $\begin{array}{l}\text { EU: impactos positivos } \\
\text { (incrementos) de los } \\
\text { precios reales del petróleo }\end{array}$ & 12.3 & 0.72 & $\begin{array}{l}\text { Dentro del círculo } \\
\text { unitario }\end{array}$ \\
\hline $\begin{array}{l}\text { EU: impactos negativos } \\
\text { (caídas) de los precios } \\
\text { reales del petróleo }\end{array}$ & 22.6 & 0.12 & $\begin{array}{c}\text { Dentro del círculo } \\
\text { unitario }\end{array}$ \\
\hline $\begin{array}{l}\text { España: impactos positivos } \\
\text { (incrementos) de los } \\
\text { precios reales del petróleo }\end{array}$ & 11.4 & 0.78 & $\begin{array}{l}\text { Dentro del círculo } \\
\text { unitario }\end{array}$ \\
\hline $\begin{array}{l}\text { España impactos negativos } \\
\text { (caídas) de los precios } \\
\text { reales del petróleo }\end{array}$ & 10.21 & 0.86 & $\begin{array}{c}\text { Dentro del círculo } \\
\text { unitario }\end{array}$ \\
\hline $\begin{array}{l}\text { Noruega: impactos de los } \\
\text { precios reales del petróleo }\end{array}$ & 13.5 & 0.63 & $\begin{array}{c}\text { Dentro del círculo } \\
\text { unitario }\end{array}$ \\
\hline $\begin{array}{l}\text { Argentina: impactos de los } \\
\text { precios reales del petróleo }\end{array}$ & 21.2 & 0.17 & $\begin{array}{c}\text { Dentro del círculo } \\
\text { unitario }\end{array}$ \\
\hline
\end{tabular}

Fuente: Elaboración propia.

Nota: Las estimaciones se realizaron con cinco retrasos en niveles (la periodicidad más uno), salvo para los modelos de EU con impactos positivos de los precios reales del petróleo y Argentina con impactos de los precios reales del petróleo, que se estimaron con seis retrasos en niveles.

\section{REFERENCIAS BIBLIOGRÁFICAS}

Akram, Farooq (2009), “Commodity Prices, Interest Rates and the Dollar”, Energy Economics 31 (6), pp. 838-851.

Amisano, Gianni, y Giannini, Carlo (1997), Topics in Structural VAR Econometrics, $2^{\mathrm{a}}$. ed., Berlin, Springer-Verlag.

Anzuini, Alessio; Lombardi, Marco, y Pagano, Patrizio (2010), "The Impact of Monetary Policy Shocks on Commodity Prices”, Есв Working Paper 1232, agosto.

Baffes, John (2007), “Oil spills on other Commodities”, Policy Research Working Paper 4333, The World Bank. 
Bjornland, Hilde (2000), "The Dynamic Effects of Aggregate Demand, Supply and Oil Price Shocks. A Comparative Study", The Manchester School, 68 (5), pp. 578607.

Blanchard, Oliver, y Galí, Jordi. (2007) "The Macroeconomic Effects of Oil Shocks: why are the 2000s so different from the 1970s?", working paper 13368, National Bureau of Economic Research, septiembre.

British Petróleum (2011), BP Statistical Review of World Energy, June 2011, London, BP Statistical Review of World Energy.

Brown, Stephen, y Yucel, Mine (1999), "Oil Prices and us Aggregate Economic Activity: a Question of Neutrality", Federal Reserve Bank of Dallas Economic and Financial Review, 1999 (segundo trimestre), pp. 16-23.

Cashin, Paul; Céspedes, Luis, y Sahay, Ratna (2004), "Commodity Currencies and the Real Exchange Rate", Journal of Development Economics, 75(1), pp. 239-268.

Cheung, Calista, y Morin, Sylvie (2007), "The Impact of Emerging Asia on Commodity Prices”, working paper 2007/55, Banco de Canadá.

Coyle, William (2007), "The Future of Biofuels. A Global Perspective", documento de trabajo, Economic Research Service, USDA.

Cuñado, Juncal, y Pérez de Gracia, Fernando (2003), "Do Oil Price Shocks Matter? Evidence from some European Countries", Energy Economics, 25(2), pp. 137-154.

Eichenbaum, Martin, y Evans, Charles (1995), "Some Empirical Evidence on the Effects of Shocks of Monetary Policy on Real Exchange Rates", Quarterly Journal of Economics, 110 (4), pp. 975-1009.

Elder, John, y Serletis, Apostolos (2010), "Oil Price Uncertainty", Journal of Money, Credit and Banking, 42 (6), pp. 985-1198.

Frankel, Jeffrey (2008), “The Effect of Monetary Policy on Real Commodity Prices”, en J. Campbell (ed.), Asset Prices and Monetary Policy, Chicago, University of Chicago Press, pp. 291-327.

Frankel, Jeffrey, y Rose, Andrew (2010), "Determinants of Agricultural and Mineral Commodity Prices", Working Paper Series rwp10-038, Kennedy School of Government, Havard University.

Gilbert, Christopher (2010), "How to understand High Food Prices", Journal of Agricultural Economics, 61 (2), pp. 398-425.

Gisser, Micha, y Goodwin, Thomas (1986), "Crude Oil and the Macroeconomy: tests of some Popular Notions", Journal of Money, Credit and Banking, 18 (1), pp. $95-103$.

Hamilton, James (1983), "Oil and Macroeconomy since World War II", Journal of Political Economy, 91 (2), pp. 228-248. 
- (2003), "What is an Oil Shock?”, Journal of Econometrics, 113 (2), pp. 363-398.

- (2009), "Causes and Consequences of the Oil Shock of 2007-08", Brookings Papers on Economic Activity, 40 (1) 215-283.

IMF (2011a), "Estadísticas Financieras Internacionales" [disquete], base de datos del Fondo Monetario Internacional.

- (2011b), World Economic Outlook, Washington, DC, International Monetary Fund.

- (2012), World Economic Outlook, Washington, DC, International Monetary Fund.

Jiménez-Rodríguez, Rebeca, y Sánchez, Marcelo (2005), “Oil Price Shocks and Real GDP Growth. Empirical Evidence for some oECD Countries”, Applied Economics, 37 (2), pp. 201-228.

Kilian, Lutz, y Hicks, Bruce (2009), "Did unexpectedly strong Economic Growth cause the Oil Price Shock of 2003-2008?”, CEPR Discussion Paper 7265, abril.

Lütkepohl, Helmut (1991), Introduction to Multiple Time Series Analysis, Berlin, Springer Verlag.

Nakaso, Hiroshi (2011), Report of the G-20 Study Group of Commodities, s.1., G-20.

Sachs, Jeffrey (1982), "The Oil Shocks and Macroeconomic Adjustment in the United States", European Economic Review, 18 (1), pp. 243-248.

Sims, Christopher (1980), "Macroeconomics and Reality", Econometrica, 48 (1), pp. 1-48.

Singleton, Kenneth (2011), "Investor Flows and the 2008 Boom/Bust in Oil Prices", working paper, Stanford Graduate School of Business, julio.

Trinh, Tamara; Voss, Silha, y Dyck, Steffen (2006), China's Commodity Hunger, Frankfurt, Deutsche Bank Research. 